\title{
A fast full-field 3D measurement system for BGA coplanarity inspection
}

\author{
H. N. Yen and D. M. Tsia \\ Machine Vision Lab. \\ Department of Industrial Engineering and Management \\ Yuan-Ze University, Chung-Li, Taiwan, R.O.C. \\ E-mail: iedmtsai@saturn.yzu.edu.tw
}

\begin{abstract}
Ball-Grid-Array (BGA) has become one of the most popular packaging techniques in the electronic industry. Coplanarity inspection of BGA solder balls is critical in BGA manufacturing for process and quality control. Currently, the 3D measurement systems for BGA coplanarity inspection are mainly based on laser scanning techniques. However, they suffer from low inspection speed due to the physical line-scanning process. In this paper, a fast and cost-effective 3D measurement system for coplanarity inspection of BGA solder balls is proposed. The proposed system uses an LCD-based phase measuring technique to perform full-field 3D measurement of BGA solder balls with high accuracy. Experiments have shown that the coplanarity measurement of BGA solder balls is very efficient and effective with the proposed system. The measurement repeatability is in the micrometer range. The processing time of the proposed 3D measurement system for an image of $640 \times 480$ pixels is less than 2 seconds on a typical personal computer.
\end{abstract}

Keywords: 3D measurement; Ball-Grid-Array; Coplanarity inspection; Phase shift; Fringe projection 


\section{Introduction}

In recent years, the trend toward light, thin, small and multifunctional electronic products has been demanding high density integrated circuit (IC) chips. Various chip-packaging techniques have been developed to meet this requirement. Among these techniques, Ball-Grid-Array (BGA) has become one of the most popular in the electronic industry because it offers higher density I/Os at a relatively lower manufacturing cost [1].

As shown in Figure 1, a BGA chip uses regularly-spaced solder balls as electric connectors to interconnect with the printed circuit board (PCB). The reliability of a BGA chip is strongly dependent on these solder balls that must be mounted to meet specifications for ball location, ball size and ball coplanarity [2] in the ball placement process. However, the ball placement process is complex, and is influenced by many process factors such as flux paste, ball acquisition, ball-to-pad alignment and ball deposition. When solder ball related defects are encountered, they induce open or short circuits and result in unsecure electric connections.

Ball defects such as missing balls, offset balls, improper size balls and bridge balls can be generally detected by using two dimensional (2D) machine vision systems [3, 4]. However, coplanarity inspection of solder balls cannot be arranged in 2D space since such inspection requires ball-height information. This calls for a three dimensional (3D) measurement system. Currently, nondestructive 3D measurement for coplanarity inspection of BGA solder balls is mainly performed by laser-based systems [5-7]. These systems use an optical triangular method by projecting a laser line onto solder balls and detecting its reflection to measure 3D data. Since only one 
line can be measured at a time, the laser head or the worktable must be physically moved to construct the whole 3D profile of solder balls. This results in slow measurement speed and the need for high-precision motion control. Any vibration occurring in the movement makes the $3 \mathrm{D}$ measurement imprecise. In addition, the measuring accuracy of laser-based systems is greatly affected by laser beam spot reflection and stray light.

In this paper, a fast and cost-effective 3D measurement system for coplanarity inspection of BGA solder balls is proposed. Different from the line-scanning measurement of traditional laser-based systems, the proposed system with its phase measuring technique can provide full-field 3D measurement of BGA solder balls. The proposed system uses an incoherent light source and a software-controlled LCD grating to project an area of fine sinusoidal fringes on the BGA solder balls under inspection. The projected sinusoidal fringes will be deformed by the surface height variations of solder balls. By detecting the deformed sinusoidal fringes, and in combination with phase shifting analysis, full-field 3D measurement of BGA solder balls in a sizable area can be acquired at the same time. Coplanarity measurement of BGA solder balls can be effectively and reliably calculated with numerous ball-height values obtained from the proposed full-field 3D measurement system.

This paper is organized as follows. Section 2 introduces the 3D measurement principle of the proposed system. Section 3 first describes the software-controlled LCD grating, the system configuration, and the system calibration. The coplanarity measurement of BGA solder balls is then discussed. Experimental results on BGA samples and the measurement repeatability of the proposed system are presented and analyzed in Section 4. This paper is concluded in Section 5. 


\section{The principle of phase measuring technique}

The proposed 3D measurement system for BGA coplanarity inspection is based on the phase measuring technique. The optical geometry of the projection and recording system is shown in Figure 2. When a sinusoidal fringe pattern is projected onto a 3D object, the surface height function of the object is mapped to a phase function of the deformed intensity fringe pattern, and can be resolved by

$$
h(x, y)=\frac{P_{o}}{2 \pi} \tan \theta_{d} \cdot\left[\phi_{r}(x, y)-\phi_{o}(x, y)\right]=\frac{P_{o}}{2 \pi} \tan \theta_{d} \cdot \Delta \phi(x, y)
$$

where $h(x, y)$ is the surface height of object at coordinates $(x, y), P_{o}$ is the period of sinusoidal grating in the reference plane, $\theta_{d}$ is the angle between the axis of detection and the reference plane, $\phi_{r}(x, y)$ is the phase value at coordinates $(x, y)$ on the reference plane, $\phi_{o}(x, y)$ is the phase value at coordinates $(x, y)$ with the presence of an object, and $\Delta \phi(x, y)$ is the phase difference between $\phi_{r}(x, y)$ and $\phi_{o}(x, y)$.

Because the values of optical parameters $P_{o}$ and $\theta_{d}$ are fixed throughout the measuring process, Eq. (1) can be simplified as

$$
h(x, y)=k \cdot \Delta \phi(x, y)
$$

where $k=\frac{P_{o}}{2 \pi} \tan \theta_{d}$. It is a constant for a given system setup, and can be acquired with a simple system calibration procedure described later in section 3.3. Calculation of the surface height $h(x, y)$ is now simplified to the determination of the phase 
difference $\Delta \phi(x, y)$ at each of the coordinates $(x, y)$ on the projection plane.

If a sinusoidal fringe pattern is projected onto a plane, its intensity image $I(x, y)$ can be expressed as

$$
I(x, y)=I_{b}(x, y)+I_{m}(x, y) \cos \phi(x, y)
$$

where $I_{b}(x, y)$ is the background intensity, $I_{m}(x, y)$ is the fringe modulation, and $\phi(x, y)$ is the phase distribution at coordinates $(x, y), I(x, y)$ in Eq. (3) is the known intensity image of the projected sinusoidal fringe pattern obtained from the sensing CCD camera. $I_{b}(x, y), I_{m}(x, y)$ and $\phi(x, y)$ are three unknown variables in Eq. (3). To extract the desired phase $\phi(x, y)$, at least three different intensity images of the projected sinusoidal fringe patterns are required.

If the phase shifting increment is $\pi / 2$ and the four-step phase shifting method is adopted, then the intensity images of sinusoidal gratings by sequential phase shifting can be formulated as

$$
\begin{aligned}
& I_{1}(x, y)=I_{b}(x, y)+I_{m}(x, y) \cos [\phi(x, y)+0]=I_{b}(x, y)+I_{m}(x, y) \cdot \cos \phi(x, y) \\
& I_{2}(x, y)=I_{b}(x, y)+I_{m}(x, y) \cos \left[\phi(x, y)+\frac{\pi}{2}\right]=I_{b}(x, y)-I_{m}(x, y) \sin \phi(x, y) \\
& I_{3}(x, y)=I_{b}(x, y)+I_{m}(x, y) \cos [\phi(x, y)+\pi]=I_{b}(x, y)-I_{m}(x, y) \cdot \cos \phi(x, y) \\
& I_{4}(x, y)=I_{b}(x, y)+I_{m}(x, y) \cos \left[\phi(x, y)+\frac{3 \pi}{2}\right]=I_{b}(x, y)+I_{m}(x, y) \sin \phi(x, y)
\end{aligned}
$$

By solving the four equations above, the phase $\phi(x, y)$ at coordinates $(x, y)$ can be computed as 


$$
\phi(x, y)=\tan ^{-1}\left[\frac{I_{4}(x, y)-I_{2}(x, y)}{I_{1}(x, y)-I_{3}(x, y)}\right]
$$

The advantage of using the four-step phase shifting method is that zero value at the denominator in Eq. (4), which may occur in the three-step phase shifting method, can be avoided. The phase $\phi(x, y)$ calculated from Eq. (4) is between $-\pi / 2$ and $\pi / 2$, and is not the actual phase value. It cannot be directly used to evaluate the surface height. With the so-called modulo $2 \pi$ correction [8], the calculated phase $\phi(x, y)$ from Eq. (4) can be extended to the desired range between 0 and $2 \pi$. For pixel coordinates $(x, y)$, the $2 \pi$ discontinuities of modulo $2 \pi$ phase data can be removed by adding $2 \pi$ to the pixel $(x, y)$ as long as the phase variation of the pixel $(x, y)$ and its previous adjacent pixel $(x-1, y)$ is larger than $\pi$, i.e.,

$$
\phi(x, y)= \begin{cases}\phi(x, y)+2 \pi & \text { if } \phi(x-1, y)-\phi(x, y)>\pi \\ \phi(x, y) & \text { otherwise }\end{cases}
$$

The phase functions on the reference plane and on the object, i.e., $\phi_{r}(x, y)$ and $\phi_{o}(x, y)$ in Eq. (2), can be obtained according to the forgoing phase unwrapping process. Once the phases that modulate the sinusoidal fringe patterns on the object and on the reference plane are obtained respectively, the phase difference $\Delta \phi(x, y)$ can be determined. Then the surface height value of each coordinate on the object can be derived from Eq. (2).

\section{System description and coplanarity measurement}

Since the proposed system is based on phase measuring technique, there are two essential parts of the technique to consider as it is applied to perform 3D measurement, 
i.e., generating the sinusoidal fringe pattern and shifting the generated fringe pattern with accurate phase increment. In the proposed system, these two critical parts are realized by using a liquid crystal display (LCD) grating. In this section, we first describe the software-controlled LCD grating and the system layout. Then a simple system calibration procedure to obtain the system constant $k$ in Eq. (2) is introduced. Finally, the coplanarity measurement of BGA solder balls with the proposed system is described.

\subsection{The software-controlled LCD grating}

Sinusoidal fringe patterns are traditionally generated by interference of two laser beams. But the speckle of noise produced by the coherent lights will affect the intensity distribution of the generated sinusoidal fringe pattern, and result in inaccurate 3D measurement. Besides by the laser interference method, sinusoidal fringe patterns have been generated by illuminating a fixed sinusoidal transparency grating with an incoherent light source. However, an accurate sinusoidal transparency grating is difficult to manufacture [9], and lacks flexibility for various objects.

The LCD panel has uniform optical transparency, and can be software-controlled by connecting it to a personal computer. The unique electro-optical capability of the LCD panel makes it well suited as a controllable sinusoidal transparency grating. In the proposed system, a sinusoidal fringe pattern can be easily created on the LCD panel with a simple setting of parameters including pattern area, fringe period and phase shifting increment. Owing to the pixel structure of an LCD panel, these control parameters are counted by pixels in the proposed system. For example, Figure 3(a) shows a computer-generated sinusoidal fringe pattern displayed on the LCD panel, in 
which the fringe period is 20 pixels. Figure 3(b) shows its sinusoidal intensity profile along the horizontal direction in 8-bit gray levels. After illuminating the created sinusoidal transparency grating with collimated incoherent light, the required sinusoidal fringe pattern in the proposed system can be easily obtained.

As aforementioned, the proposed system adopts the four-step phase shifting method with $\pi / 2$ phase shifting increment (i.e., one fourth of a fringe period). Therefore, accurate phase shifting can be realized in the proposed system by simply setting the fringe period to a multiple of four. The phase shifting process is conducted by a software-controlled process instead of a physical movement process. This approach is superior to the traditional methods [10-13] that generally perform inaccurate phase shifting by mechanical movements of the slide projector or piezocrystal (PZT). The measuring error due to the vibration of mechanical movement and inaccurate phase shifting in convectional methods can be effectively avoided in the proposed system.

\subsection{System layout}

The configuration of the proposed 3D measurement system for BGA solder balls inspection is shown in Figure 4. The most important part in this system is the LCD panel, which is of $800 \times 600$ pixels resolution and has an active area of $27 \times 20 \mathrm{~mm}^{2}$. This panel is connected to a personal computer, and can be directly controlled by software to generate a transparent sinusoidal grating through an LCD control interface. Since the transparency variation of an LCD panel will be affected by high temperature [14], a cool light source with a power range of 0-150 Watts and a fiber optic light guide are adopted in the system to reduce the transparency variation of the LCD panel. 
A collimated lens (Lens 1 in Figure 4) with a focal length of $50 \mathrm{~mm}$ is set up in front of the cool light source to produce collimated light. The collimated light is used to illuminate the software-controlled LCD grating to project the uniform sinusoidal fringe pattern onto the object or the rigid flat plate (The rigid flat plate is used as a reference plane).

A projected lens (Lens 2 in Figure 4) with focal length of $50 \mathrm{~mm}$ is used to focus the images of the projected sinusoidal fringe pattern onto the rigid flat plate. A precision translation stage with resolution of $0.01 \mathrm{~mm}$ is used to hold the rigid flat plate and to place the BGA chip under inspection. The collimated lens, the LCD panel, the projected lens and the precision translation stage are all held by optical holders and fixed on a linear optical bench $50 \mathrm{~cm}$ in length.

The projected fringe pattern is captured by a CCD camera equipped with a $50 \mathrm{~mm}, F / 1.8$ collocated lens. The CCD camera captures the intensity images from an inclined detection angle of 20 degrees. The sensed image is of $640 \times 480$ pixels with 8-bit gray levels.

\subsection{System calibration}

For a given system setup, the constant value $k$ in Eq. (2) must be determined prior to the inspection process. It can be seen from Eq. (2) that constant $k$ can be derived from a known surface height divided by its corresponding phase difference. A simple calibration procedure based on a uniform measurement scale [15] is used to obtain the value $k$. The calibration procedure is described as follows. 
First, the phase distribution on the flat plate is determined by the proposed system. Then the precision translation stage that holds the flat plate is fine-tuned along the $\mathrm{z}$ direction with a known distance $\Delta d$ (used as the constant surface height $h)$. Finally, the phase distribution on the flat plate after the movement $\Delta d$ is determined again by the proposed system. Following the calibration procedure described above, the phase change $\Delta \phi$ due to a translation of the flat plate by a known distance $\Delta d$ is measured using Eq. (4). Therefore, the value $k$ can be obtained as

$$
k=\frac{\Delta d}{\Delta \phi}
$$

The adopted calibration procedure in the proposed system has the advantage that the aberrations of the optical lens and the position of the CCD camera are not necessary to be known and have no effect on the measurement accuracy.

\subsection{Ball Coplanarity measurement}

In order to inspect the coplanarity of the BGA solder balls, the height value of each solder ball must be determined first. In the proposed system, the height of a BGA solder ball is calculated from the average of $3 \times 3$ neighborhood surface height values of the central point in each solder-ball area, i.e.,

$$
H_{i}(x, y)=\frac{1}{9} \sum_{m=-1}^{1} \sum_{n=-1}^{1} h\left(x_{i}+m, y_{i}+n\right)
$$

where $h\left(x_{i}, y_{i}\right)$ is the surface height value of the central point in each solder-ball area. The coplanarity of BGA solder balls is then calculated from these ball-height 
values by the formula below [6]

$$
\operatorname{Cop}=\left[\frac{1}{N-1} \sum_{i=1}^{N}\left(H_{i}-\bar{H}\right)^{2}\right]^{1 / 2}
$$

where $N$ is the total number of solder balls under inspection, $H_{i}$ represents the height value of the $i$-th solder ball, and $\bar{H}$ is the mean height value of $H_{i}$.

\section{Experimental Results}

In this study, two sample BGA chips are used to evaluate the efficacy of the proposed 3D measurement system for BGA coplanarity inspection. The measuring area of the first BGA chip (sample 1) is marked by the white-dotted frame in Figure 5(a). A sinusoidal fringe pattern of $10 \times 10 \mathrm{~mm}$ is projected on the measuring area that contains 16 BGA solder balls. Figure 5(b) shows the deformed fringes image. Figure 5(c) shows the range image as an intensity function, in which brightness is proportional to the magnitude of surface height. Figure 5(d) shows the 3D surface profile of the solder balls on the BGA substrate. To evaluate system repeatability, one of the BGA solder balls under inspection was measured twenty times by the proposed system. An average of $0.576 \mathrm{~mm}$ with standard deviation $0.0025 \mathrm{~mm}$ is obtained by the proposed system. For comparison purposes, this solder ball was also measured by a laser-based 3D measurement system, and the measuring value was $0.572 \mathrm{~mm}$. Coplanarity measurement of the solder balls on sample-1 BGA chip is $0.028 \mathrm{~mm}$. This coplanarity value is much smaller than $0.15 \mathrm{~mm}$, which is the industrial standard laid down by JEDEC (Joint Electron Device Engineering Council) for secure connection with PCBs [16]. 
The 3D measurement of the second BGA chip (sample 2) was performed under the same conditions as for the sample-1 BGA chip. The measuring area of sample-2 BGA chip is marked by the white-dotted frame in Figure 6(a). Within the measuring area, there are 48 solder balls and a missing-ball defect. Generally, the missing-ball defect cannot be easily identified from the $2 \mathrm{D}$ intensity image because of the light reflection of solder pad. Using the proposed 3D measurement system, the missing-ball defect can be clearly identified from the resulting range image or the $3 \mathrm{D}$ surface profile, as shown in Figure 6(c) and Figure 6(d). Coplanarity measurement of the solder balls on sample-2 BGA chip is $0.417 \mathrm{~mm}$, which far exceeds $0.15 \mathrm{~mm}$ due to the effect of the missing ball. This distinctly high coplanarity value, compared to the coplanarity of sample 1, indicates that an insecure connection will happen when the BGA chip is interconnected with PCBs.

The superiority of the proposed system over the laser-based systems on the 3D measurement can also be demonstrated by the above measuring data. It is observed that the number of solder balls in sample 2 (Figure 6) is three times of those in sample 1 (Figure 5). If the 3D measurements of these two samples are performed by laser-based systems, the measuring time for sample 2 will be distinctly longer than for sample 1. However, the measuring time is the same in the experiments. The reason is that the proposed system performs 3D measurement with an area-measuring scheme. The 3D data are acquired simultaneously within the area of the projected sinusoidal fringe pattern. Therefore, the measuring time will be the same provided that the projected sinusoidal fringe pattern is fixed for the test samples. This is the merit of the proposed full-field 3D measurement system. In the experiments, the processing time of the proposed 3D measurement for an image of $640 \times 480$ pixels is less than 2 
seconds on a Pentium III 500-MHz personal computer.

\section{Conclusions}

In this paper, a fast full-field 3D measurement system for coplanarity inspection of BGA solder balls is proposed. The proposed system uses an LCD-based phase measuring technique and can perform 3D measurement of BGA solder balls in an area. This area-measuring approach is superior to the line-scanning scheme of laser-based systems developed for BGA solder balls inspection. The proposed system integrates a software-controlled LCD grating and an incoherent light source to generate the required sinusoidal fringe pattern and to obtain accurate shift phase. It avoids measuring errors from the laser speckle effect and inaccurate phase shifting in BGA coplanarity inspection applications, compared to the laser-based inspection systems and the traditional phase shifting techniques. Experiments from real samples of BGA solder balls have shown that the $3 \mathrm{D}$ measurement process by the proposed system is efficient and effective. The proposed system is easily implemented and fast for coplanarity inspection of BGA solder balls. 


\section{References}

1. J. H. Lau, Ball Grid Array Technology, McGraw-Hill, New York, 1995.

2. J. Miremadi, Impact of PBGA-ball-coplanarity on formation of solder joints, Proceedings of Electronic Components and Technology, 45, pp. 1039-1050, 1995.

3. K. W. Ko, J. W. Kim, H. S. Cho, K. S. Jin and K. I. Ko, Enhancement of placement accuracy for SMD via development of a new illumination system, Proceedings of SPIE, 4190, pp. 51-61, 2001.

4. Caracappa, Ed(RVSI Acuity CiMatrix), Machine vision inspection of BGAs, SMT Surface Mount Technology Magazine, 12, pp. 48-49, 1998.

5. D. J. Svetkoff, D. K. Rohner, D. A. Nohleti and R. L. Jackson, Method and system for triangulation-based, 3-D imaging utilizing an angled scanning beam of radiant energy, U.S. Patent 5617209, April, 1997.

6. P. Kim, and S. Rhee, Three-dimensional inspection of ball grid array using laser vision system, IEEE Transactions on Electronics Packaging Manufacturing, 22, pp. 151-155, 1999.

7. C. Sun, H. Shi, Y. Oiu and S. Ye, Line-structured laser scanning measurement system for BGA lead coplanarity, Circuits and Systems, The 2000 IEEE Asia-Pacific Conference, pp. 715 -718, 2000.

8. J. E. Greivenkamp and J. H. Bruning, Phase shifting interferometry, in Optical Shop Testing, D. Malacara (ed.), Wiley, New York, 1992.

9. T. Xian and X. Su, Area modulation grating for sinusoidal structure illumination on phase-measuring profilometry, Applied Optics, 40, pp. 1201-1206, 2001.

10. V. Srinivasan, H. C. Liu and M. Halioua, Automated phase-measuring profilometry of 3-D diffuse objects, Applied Optics, 23, 3105-3108, 1984.

11. S. Toyooka and Y. Iwaasa, Automatic profilometry of 3-D diffuse objects by spatial phase detection, Applied Optics, 25, pp. 1630-1633, 1986.

12. V. Srinivasan, H. C. Liu and M. Halioua, Automated phase-measuring profilometry: a phase mapping approach, Applied Optics, 24, 185-188, 1985.

13. G. Mauvoisin, F. Bremand, and A. Lagarde, Three-dimensional shape reconstruction by phase shifting shadow moiré, Applied Optics, 33, pp. 2163-2169, 1994.

14. F. Docchio, G. Sansoni and N. Viviani, Light-induced transmission changes in 
liquid crystal displays for adaptive pattern projection, IEEE Transactions on Instrumentation and Measurement, 41, pp. 629-632, 1992.

15. A. Asundi and W. Zhou, Unified calibration technique and its applications in optical triangular profilometry, Applied Optics, 38, pp. 3556-3561, 1999.

16. D. Edwards, Development of JEDEC standard thermal measurement test boards, IEEE Transactions on Components, Packaging and Manufacturing Technology, Part A, 19, pp. 478-485, 1996. 


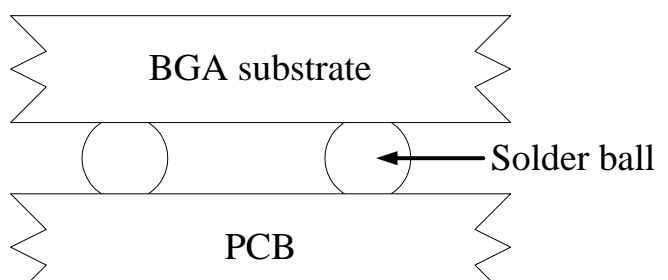

Fig. 1. Interconnection between a BGA chip and a PCB.

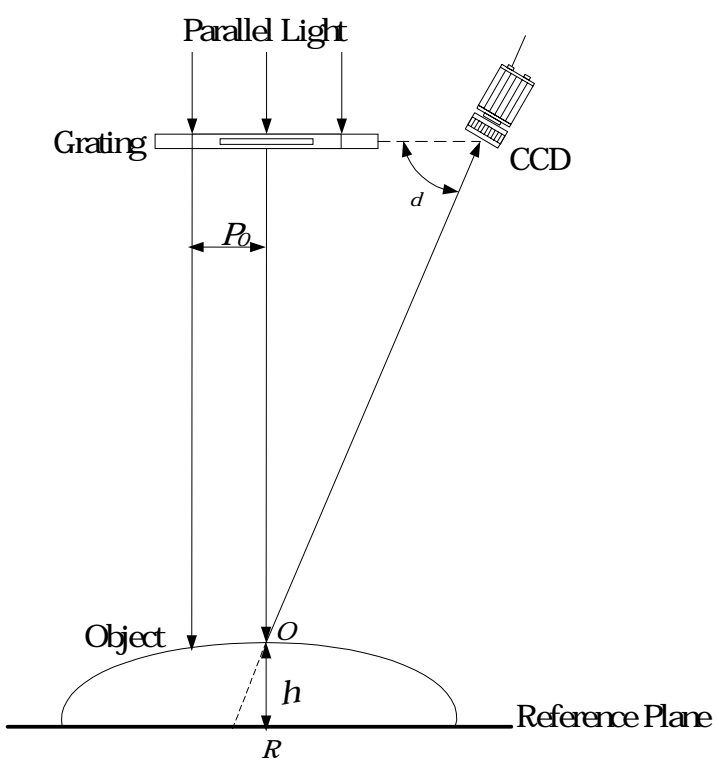

Fig. 2. Optical geometry of the projection and recording system. 


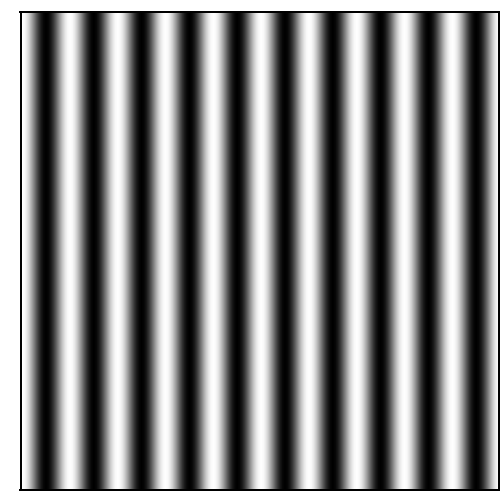

(a)

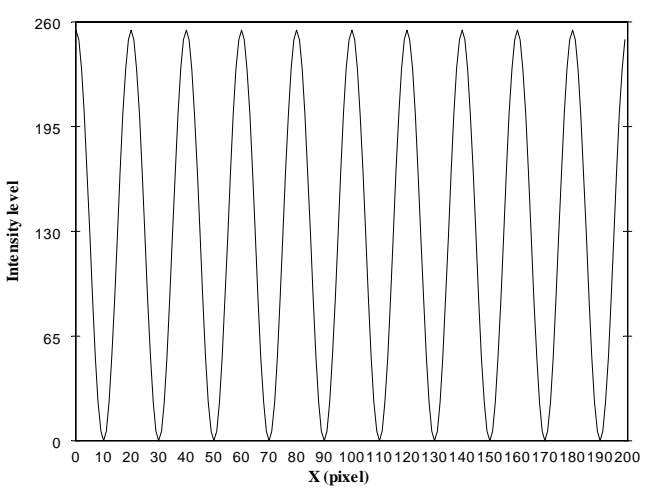

(b)

Fig. 3. (a) An example of computer-generated sinusoidal fringe pattern;

(b) the corresponding intensity profile in 8-bit display of a horizontal line in (a).

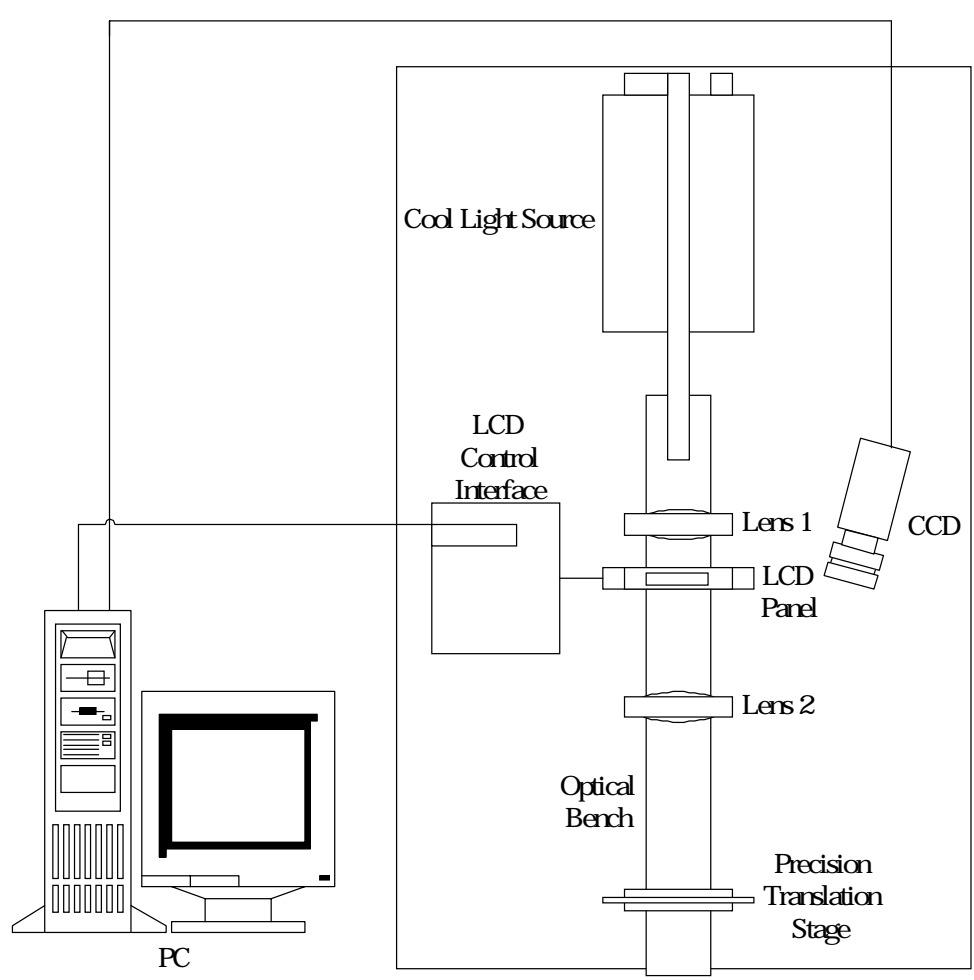

Fig. 4. Configuration of the proposed 3D measurement system. 


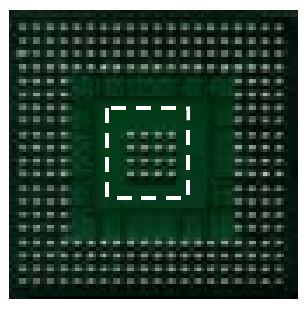

(a)

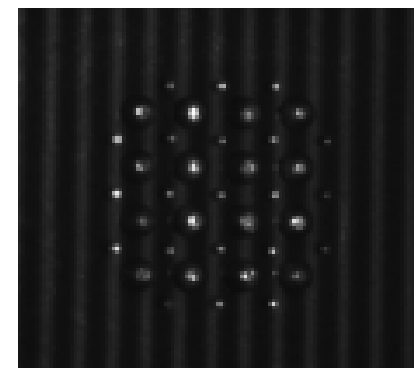

(b)

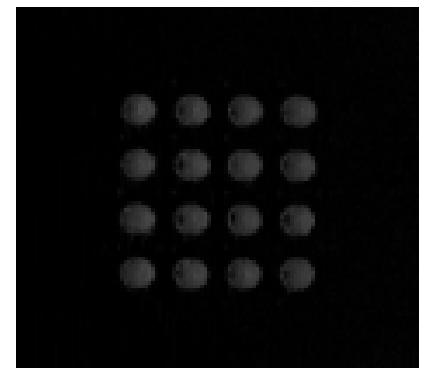

(c)

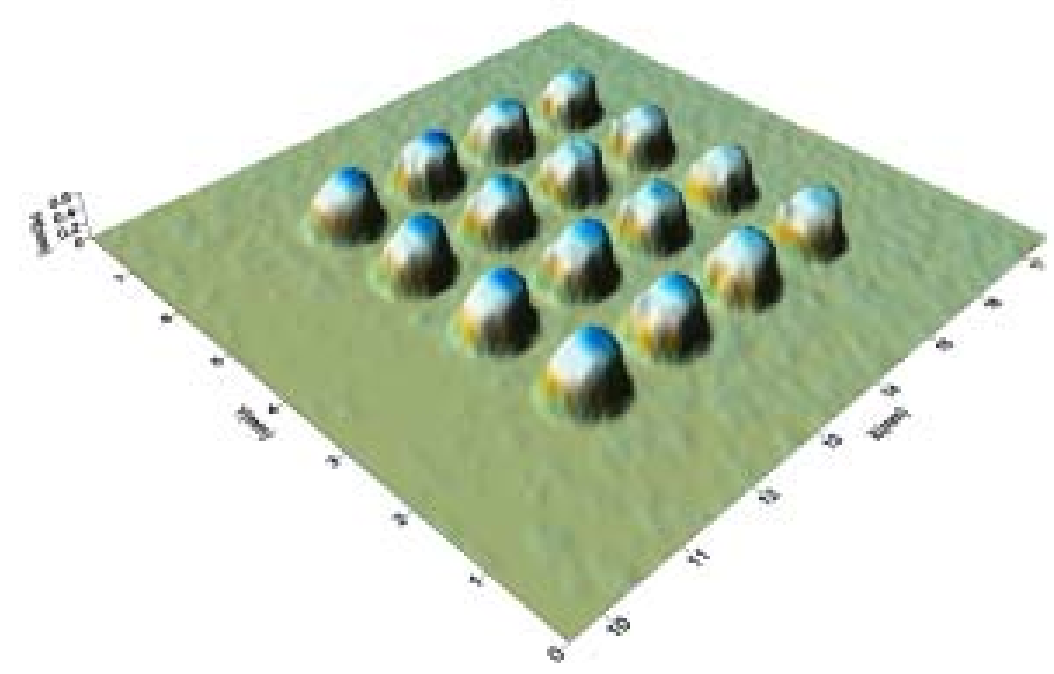

(d)

Fig. 5. Sample 1 of BGA solder balls: (a) original image; (b) deformed image; (c) range image as an intensity function; (d) 3D surface profile. 


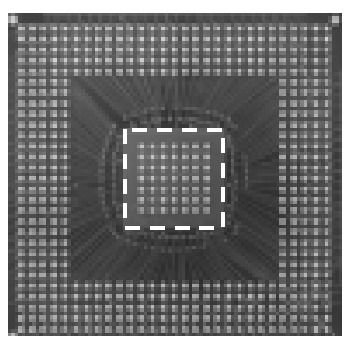

(a)

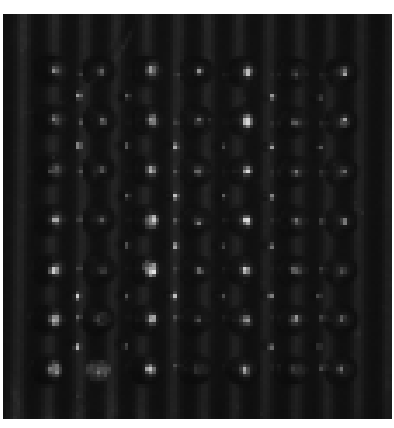

(b)

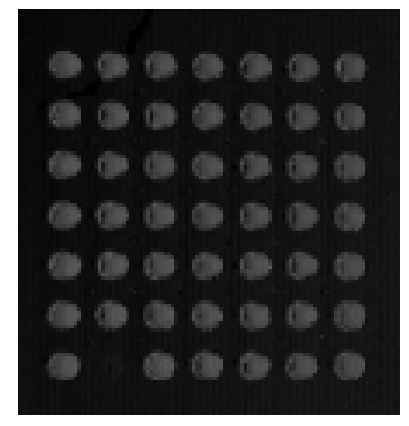

(c)

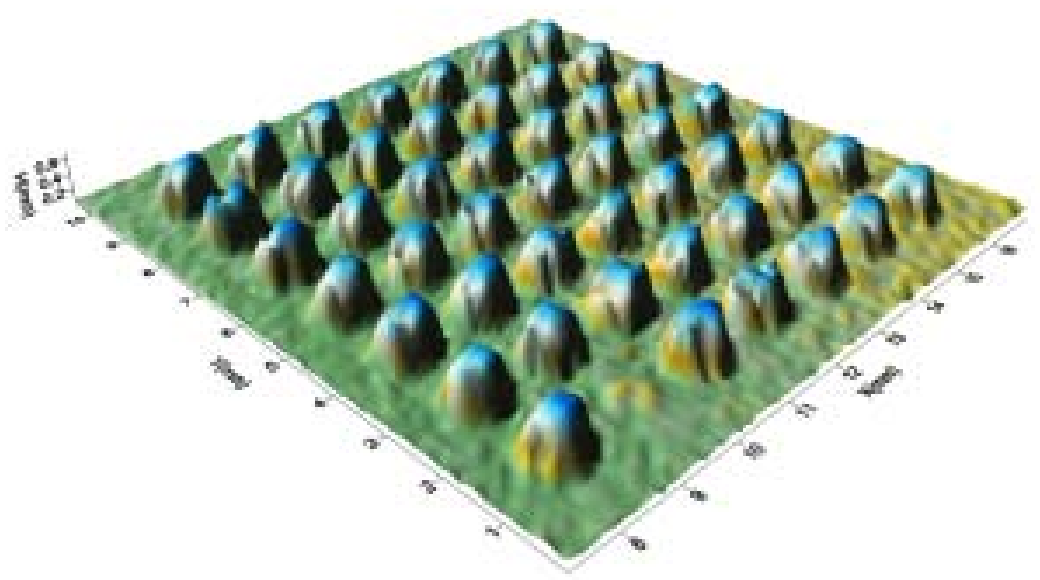

(d)

Fig. 5. Sample 2 of BGA solder balls: (a) original image; (b) deformed image; (c) range image as an intensity function; (d) 3D surface profile. 